\title{
A novel lineage of polyomaviruses identified in bark scorpions
}

3 Kara Schmidlin ${ }^{1,2}$, Simona Kraberger ${ }^{1}$, Chelsea Cook $^{3}$, Dale F. DeNardo², Rafaela S. 4 Fontenele ${ }^{1,2}$, Koenraad Van Doorslaer ${ }^{4,5}$, Darren P. Martin' ${ }^{6}$, Christopher B. Buck ${ }^{7}$, Arvind 5 Varsani $1,2,8,9^{*}$

61 The Biodesign Center for Fundamental and Applied Microbiomics, Arizona State University, 7 Tempe, Arizona, AZ 85287, USA

82 School of Life Sciences, Arizona State University, Tempe, Arizona, AZ 85287, USA

$9{ }^{3}$ Department of Biological Sciences, Marquette University, 11428 W. Clybourn St., Milwaukee, 10 WI 53233, USA

$11{ }^{4}$ Genetics Graduate Interdisciplinary Program, University of Arizona, Tucson, AZ 85719, USA;

$125^{5}$ School of Animal and Comparative Biomedical Sciences, The BIO5 Institute, Department of 13 Immunobiology, Cancer Biology Graduate Interdisciplinary Program, UA Cancer Center, 14 University of Arizona Tucson, Tucson, AZ 85724, USA

$15{ }^{6}$ Computational Biology Division, Department of Integrative Biomedical Sciences, Institute of 16 Infectious Diseases and Molecular Medicine, University of Cape Town, Observatory, Cape Town 17 7925, South Africa

$18{ }^{7}$ Lab of Cellular Oncology, National Cancer Institute, National Institutes of Health, Bethesda, USA

$19{ }^{8}$ Center for Evolution and Medicine, Arizona State University, Tempe, Arizona, AZ 85287, USA

209 Structural Biology Research Unit, Department of Clinical Laboratory Sciences, University of 21 Cape Town, 7925, Cape Town, South Africa

* Corresponding author: Arvind Varsani - Arvind.varsani@asu.edu

GenBank Accession \#s: MW175892-MW175899 


\section{Abstract}

Polyomaviruses are non-enveloped viruses with circular double-stranded DNA genomes that range in size from 4-7 kilobasepairs. Initially identified in mammals, polyomaviruses have now been identified in birds and a few fish species. Although fragmentary polyomavirus-like sequences have been detected as apparent 'hitchhikers' in shotgun genomics datasets for various arthropods, the possible diversity of these viruses in invertebrates remains unclear. In general, polyomaviruses are host-specific, showing strong evidence of host-virus co-evolution. Identification of polyomaviruses in a broader range of animals could shed useful light on the evolutionary history of this medically important group of viruses. Scorpions are predatory arachnids that are among the oldest terrestrial animals. Thus far, viromes of arachnids have been under-sampled and understudied. Here, high-throughput sequencing and traditional molecular techniques were used to explore the diversity of circular DNA viruses associated with bark scorpions (Centruroides sculpturatus) from the greater Phoenix area, Arizona, USA. The complete genomes of eight novel polyomaviruses were identified. Analysis of Centruroides transcriptomic datasets elucidated the splicing of the viral late gene array, which is more complex than that of vertebrate polyomaviruses. Phylogenetic analysis provides further evidence of codivergence of polyomaviruses with their hosts, suggesting that at least one ancestral species of polyomaviruses was circulating amongst the primitive common ancestors of arthropods and chordates.

\section{Introduction}

Scorpions are one the oldest terrestrial arthropods, with fossil records dating back 450 million years. They are venomous, predatory animals that belong to the class Arachnida. Nearly 2200 scorpion species are recognized, occurring in a wide range of ecosystems including deserts, grasslands, savannahs, deciduous forests, pine forests, intertidal zones, rain forests and caves (1). Their bodies are separated into two segments, a cephalothorax and an abdomen that includes the tail and stinger, four pairs of legs and pedipalps with plier-like pinchers (2). Bark scorpions (Centruroides sculpturatus) are nocturnal, generalist predators that consume a wide variety of insects, spiders, centipedes and other scorpions. They inhabit arid desert and riparian regions in south-western North America $(2,3)$. Although these scorpions are desert adapted, water is a limiting factor, so they are most commonly found in riparian areas. The bark scorpion is a common 
62 The first polyomavirus was discovered in the mid-1950s as a filterable agent and was shown to

63 induce multiple classes of tumors when experimentally injected into animals, suggesting the name

64 poly (multiple) oma (tumors). Polyomaviruses have been recovered from a variety of mammals

65 including humans, bats, and rodents, and diverse polyomavirus species have recently been

66 reported in an assortment of birds and fish $(5,6)$.

67 Polyomaviruses have a conserved genome organization that consists of an early region and a 68 late region separated by a regulatory region (RR) that encompasses the early and late promoters 69 as well as the origin of replication. Polyomavirus genomes are remarkably simple, encoding only 70 three absolutely essential proteins. The Large Tumor antigen (LT) is a multipurpose protein that 71 encodes a superfamily 3 helicase domain that unwinds the origin of replication and primes the 72 host cell DNA replication machinery to replicate the viral genome. LT also encodes a DNAJ-like 73 domain, which encodes a hallmark HPDKGG motif, and a pRB-interaction motif, LXCXE (7). All 74 known polyomavirus late regions encode a major capsid protein, VP1, which forms the non75 enveloped icosahedral surface of the virion, and a minor capsid protein, VP2, that associates with 76 the inner surface of the assembled virion. A hallmark feature of VP2 is an N-terminal 77 myristoylation motif. (7).

78 Although polyomaviruses are host specific, strict virus-host co-divergence models do not fully explain polyomavirus evolution. A growing body of evidence suggests an ancient and relatively stable association of polyomaviruses with their hosts (5). While this long-standing association implicates co-divergence as the main driver of polyomavirus evolution, there are deviations that indicate additional factors may occasionally contribute to their evolutionary history including switching between closely related animal hosts, intra-host lineage duplication followed by divergence (i.e., viral speciation within the same host species), and incomplete lineage sorting (persistence of viral polymorphisms during successive host divergence events) (5). Viral metagenomics and molecular techniques, such as degenerate PCR and random-primed rollingcircle amplification (RCA), are enabling the identification and recovery of polyomaviruses from novel hosts at an increased frequency. This has allowed for a deeper understanding of polyomavirus evolutionary history. Shotgun deep sequencing has also begun revealing viral sequences as inadvertent contaminants in genomics and transcriptomics studies. Since shotgun genomics studies are not designed to enrich for viruses and do not require careful management of sample cross-contamination, these adventitious virus sequences can be difficult to interpret.

93 For example, although a prior data mining effort detected highly divergent polyomavirus-like 94 sequences in a shotgun genomics dataset for California bark scorpion, it is difficult to rule out the 
possibility that the sequence came from an ectoparasite or environmental source, as opposed to a productive infection of the scorpion itself (5). Because the ultimate sources of data-mined sequences are uncertain, directed discovery of additional polyomavirus sequences in invertebrates could help further resolve the evolutionary history of this group. This information will help generate a taxonomic framework that can be utilized to accurately categorize novel viruses in such a way that sequence similarities could be used by researchers and clinicians to aid in predicting hosts, tissue tropism and disease states.

102 The International committee for the taxonomy of viruses (ICTV) recommends using phylogeny103 based taxonomy of LT protein sequences to classify polyomaviruses into four recognized genera 104 as well as several unassigned species $(8,9)$. The four established genera are 105 Alphapolyomavirus, Betapolyomavirus and Deltapolyomavirus which contain species that infect 106 mammalian hosts and Gammapolyomavirus which contains species that infect avian hosts. 107 Recently identified polyomaviruses associated with fish datasets $(5,6,10)$ have not yet been 108 assigned to a genus. In the present report we identify and highlight the diversity of eight complete 109 polyomavirus genomes recovered from the viscera of carefully cleaned bark scorpion carcasses 110 and propose a taxonomic assignment for the group.

\section{Material and methods}

\section{Sample collection and preparation}

113 Four bark scorpions were collected from two locations, Phoenix $(n=3)$ and Tempe $(n=1)$, Arizona, 114 USA, between August and November 2018 and stored at $-80^{\circ} \mathrm{C}$. Scorpions were surface 115 sterilized by washing in $95 \%$ ethanol for 15 seconds followed by a wash in $10 \%$ bleach for 60 116 seconds before rinsing three times in pure water. The specimens were allowed to air dry in a petri 117 dish lined with sterile filter paper in a laminar flow hood prior to dissection. The stinger was 118 removed and a sterile scalpel used to cut along the side of the body in between the legs and body 119 plate. Sterile forceps were used to remove the top plate so the internal cavity was visible. The gut 120 and liver were removed and each was placed in a sterile $1.5 \mathrm{ml}$ tube and homogenized in $500 \mu \mathrm{l}$ 121 of $\mathrm{SM}$ buffer (50 mM Tris- $\mathrm{HCl}, 10 \mathrm{mM} \mathrm{MgSO}_{4}, 0.1 \mathrm{M} \mathrm{NaCl}, \mathrm{pH}$ 7.5). The homogenate was 122 processed as previously described in (11). Viral DNA was extracted using the Roche high pure 123 viral nucleic acid kit (Roche, USA) according to manufacturer's instructions and circular DNA was 124 enriched by rolling circle amplification (RCA) with TempliPhi ${ }^{\mathrm{TM}}$ amplification kit (GE Healthcare, 125 USA). 


\section{High throughput sequencing, de novo assembly and recovery of viral genomes}

127 The RCA products were used to prepare Illumina sequencing libraries which were sequenced on

128 an Illumina HiSeq4000 sequencer ( $2 \times 100$ bp paired end library) and the reads were de novo 129 assembled using SPAdes 3.12 (12). Contigs of >1000 nts were analyzed using BLASTx (13) 130 against a local viral protein sequence database. Eight de novo assembled contigs were found to 131 have sequence similarities to dsDNA viruses from the family Polyomaviridae.

132 Based on the contigs, three sets of abutting primers were designed (Table 1) to recover the 133 complete circular viral genomes by PCR. For each PCR amplification, $1 \mu \mathrm{l}$ of RCA product was 134 used as a template with specific primer pairs and Kapa Hifi Hotstart Ready Mix (Kapa Biosystems, 135 USA) using the following thermal cycling protocol: initial denaturation at $95^{\circ} \mathrm{C}$ for 3 min followed 136 by 25 cycles $98^{\circ} \mathrm{C}$ for $20 \mathrm{~s}, 60^{\circ} \mathrm{C}$ for $15 \mathrm{~s}, 72^{\circ} \mathrm{C}$ for $6 \mathrm{~min}$, final elongation at $72^{\circ} \mathrm{C}$ for 6 min and 137 a final renaturation at $4^{\circ} \mathrm{C}$ for $10 \mathrm{~min}$. The resulting $\sim 5 \mathrm{~kb}$ amplicons were resolved on a $0.7 \%$ 138 agarose gel, excised, gel purified, ligated into pJET1.2 vector (ThermoFisher Scientific, USA) and 139 transformed into XL1 Blue Escherichia coli competent cells. The resulting recombinant plasmids 140 were Sanger sequenced at Macrogen Inc. (South Korea) by primer walking. The Sanger 141 sequence contigs were assembled using Geneious Prime 2019.1.1 (Biomatter Ltd, New Zealand).

\section{Phylogenetic analysis}

143 A curated dataset that includes representative sequences of all currently known polyomavirus 144 species was downloaded on $10^{\text {th }}$ December, 2020 from 145 https://ccrod.cancer.gov/confluence/display/LCOTF/Polyomavirus and used in all proceeding 146 analyses. A maximum-likelihood of the aligned genomes of the polyomaviruses identified in this 147 study together with two assembled polyomaviruses (LN846618 LN846619) from SRA 148 SRS561933 using GTR+G substitution matrix. A maximum-likelihood phylogenetic tree was 149 constructed using the LT amino acid sequences from eight scorpion-derived polyomavirus 150 genomes from this study as well as those from LN846618-LN846619 together with those from the 151 curated dataset and also those available in the public database GenBank as part of 152 PRJNA642917 (SAMN13619660) and PRJDB7092 (SAMD00127334) from African social spider 153 and Orb-weaving spider, respectively. We also included the LT-like sequence of circular molecule 154 MH545547 (14) and that of bandicoot papillomatosis carcinomatosis viruses 1 and 2 (EU069819 155 and KP768176). The dataset was aligned using MAFFT (15) and a ML phylogenetic tree inferred 156 using PHYML (16) with the model $L G+G+I$ as determined by ProtTest (17). Branches with $<0.8$ 
157 aLRT branch support were collapsed using TreeGraph 2 (18) and the phylogenetic trees were 158 visualized using IToL (19).

\section{Pairwise identities}

Whole genome nucleotide sequences as well as LT and VP1 amino acid pairwise identities were determined using sequence demarcation tool (SDT) version $1.2(20)$.

\section{Recombination analysis}

The genomes of the arachnid polyomaviruses were aligned using MAFFT and this alignment was used to detect evidence of recombination using RDP5 (21) with default settings. Detected recombination signals with an associated $p$-value $<0.05$ for three or more of the seven recombination detection methods implemented in RDP5 were accepted as plausible evidence of

167 recombination.

\section{Results and Discussion}

\section{Genome organization and gene content}

170 Four bark scorpions were collected in Phoenix $(n=3)$ and Tempe $(n=1)$, Arizona, dissected and 171 analyzed for the presence of polyomaviruses. From three of the samples, eight complete 172 genomes generally consistent with the organization of known polyomaviruses were recovered 173 (Figure 1). Classic mammalian and avian polyomaviruses range from 4.7 to $5.4 \mathrm{~kb}$ in length. The 174 scorpion polyomavirus genomes, in contrast, range from 5420 to 5945 bp. HHPred $(22,23)$ 175 analyses and scans for hallmark linear motifs revealed open reading frames (ORFs) encoding 176 homologs of LT, VP1 and VP2. The LT ORF, which lacks an ATG initiation codon, encodes an N177 terminal DNAJ-like domain, an LXCXE motif, an endonuclease-like origin-binding domain, and a 178 superfamily 3 helicase domain. Many polyomaviruses encode an alternative LT ORF (ALTO) 179 protein overprinted in the $L T+1$ frame (24). A majority of the scorpion polyomaviruses encode an 180 ALTO-like ORF. Well-studied mammalian polyomaviruses encode an Agno protein just upstream 181 of VP2. In avian polyomaviruses the protein is incorporated into virions and is known as VP4 (25). 182 Each of the scorpion polyomavirus genomes encodes a syntenic candidate Agno ORF. The VP2 183 ORF encodes a predicted N-terminal myristoylation signal (26) and HHPRED analyses suggest 184 predicted C-terminal structural similarity to bacteriophage holin proteins. The central portion of 185 the inferred VP1 ORF shows high-confidence hits for known polyomavirus VP1 structures. 
The late regions of the scorpion polyomaviruses also show several unrecognizable ORFs. To better understand the expression of these proteins we performed data mining on available transcriptomics datasets for scorpions. A complete polyomavirus genome was assembled from RNAseq dataset SRR5958578 and was used to map introns. It was also possible to map a set of bark scorpion transcripts from BioProject PRJNA422877 to the previously data-mined WGSassembled sequence LN846618. Although neither of the transcriptomic datasets had deep coverage of the early region, clear introns were identified in late region transcripts. The mapping revealed spliced transcripts encoding four possible isoforms of Agno, a C-extended VP1, and a protein with a predicted coiled coil structure that we name VP+. An additional ORF, which we name "downstream Agno" (Dagno), is overprinted in the +1 frame of VP+ (Figure 1). It seems conceivable that the more elaborate set of late proteins observed in the scorpion polyomaviruses might enable construction of a slightly larger virion, consistent with the larger genomes of these viruses.

\section{Similarity comparison}

200 In bark scorpion carcass \#1, four polyomaviruses were identified from the gut $(\mathrm{n}=2)$ and liver $201(n=2)$. The two from the gut share 99\% genome-wide identity and the ones from the liver share $20274 \%$ identity. Overall the four polyomavirus genomes share 73-99\% identity (Figure 1). These AZ bark scorpion polyomaviruses share $73-90 \%$ genome-wide identity with the Baja California bark scorpion polyomavirus 1 (LN846619) originally assembled from WGS data (5).

205 In bark scorpion carcass \#3, a total of three genomes were recovered from the gut $(\mathrm{n}=2)$ and liver $206(n=1)$. These share 72-100\% genome-wide identity (Figure 1) in which $100 \%$ identical isolates were recovered from both tissue types. In AZ bark scorpion \#2 only one genome was identified and it is $100 \%$ identical to the one identified in bark scorpion carcass \#3 (from the liver and gut).

Official polyomavirus taxonomy guidelines (9) specify that polyomaviruses that share $<85 \%$ LT pairwise nucleotide identity should be considered to belong to distinct viral species. By this

211 standard, genomes recovered from bark scorpions can be assigned to three unique species 212 (Figure 2). Polyomavirus sequences recovered from the gut and liver of bark scorpion samples $213 \# 1$ and \#3, along with the original bark scorpion LN846619, represent species 1. Polyomavirus 214 sequences that represent species 2 were recovered from bark scorpion samples \#2 and \#3, from 215 both the liver and gut. Polyomavirus species 3 is comprised of sequences from bark scorpion \#1 216 with isolates from both liver and gut tissues (Figure 2). The fact that identical or nearly identical 
217 viruses were recovered from necropsied tissue of multiple scorpions indicates that these viruses

218 are endemic to these animals.

219 At the nucleotide level, the scorpion polyomaviruses share between 55 and $61 \%$ whole genome 220 pairwise identity with all non-arachnid polyomaviruses. LT protein sequences of the scorpion 221 polyomaviruses share between 21 and 35\% amino acid pairwise identity with all other 222 polyomaviruses and $>80 \%$ identity amongst themselves. VP1 protein sequences show even 223 higher diversity sharing only $19-31 \%$ identity with all other polyomavirus, $>56 \%$ identity with each 224 other (Figure 2).

\section{Phylogenetic analysis of $L T$}

226 A maximum likelihood phylogenetic tree was constructed from LT amino acid sequences (5). 227 Sequences included in this analysis were available prior to December 2020 and included putative 228 LT sequences from a guitarfish polyomavirus, as well as LT-bearing polyomavirus/papillomavirus 229 chimeric viruses found in bandicoots $(27,28)$. The phylogenetic tree (Figure 3 ) shows distinct 230 clades that are generally consistent with expected phylogenetic placement of their host species 231 within broad animal phylogeny. It was possible to completely collapse the polyomavirus clades 232 derived from placental mammals and avians since these were monophyletic (with the exception 233 of two divergent bat-associated polyomavirus genomes) (Figure 3). The clades containing fish234 derived polyomaviruses branched basal to the mammalian and avian polyomavirus clades and 235 roughly reflect the genetic diversity and evolutionary relationships of the fish hosts. Importantly, 236 the scorpion derived sequences cluster together and branch from a location within the 237 polyomavirus tree that might conceivably be the root of the tree. Furthermore, LT-like sequences 238 have been identified in African social spider, giant hose spider, and orb-weaver spider that cluster 239 with the scorpion polyomavirus LTs, suggesting a broad arachnid-specific clade (Figure 3).

\section{Recombination analysis}

241 Two potential recombination events were detected in two of the scorpion polyomavirus sequences 242 (summarized in Figure 4). Both the recombinant regions were identified in the LT. In the case of 243 MW175893 (from the gut of the AZ bark scorpion \#1), a recombinant region spanning 1031 nts 244 appears to have been derived from a parental virus most closely resembling MW175895; a virus 245 identified in the liver of the AZ bark scorpion \#1. Whereas in the case of MW175897 (from the gut 246 of the AZ bark scorpion \#1) a much smaller, 64 nt long, recombinant region appears to have been 
247 derived from a virus most closely resembling MW175892, MW175893 and MW175895 (all of

248 which were identified from AZ bark scorpion \#1).

\section{Concluding remarks}

250 Eight polyomavirus genomes were recovered from three bark scorpions collected in urban 251 backyards in Phoenix and Tempe, Arizona (USA). Four polyomavirus genomes were recovered 252 from the livers and four from the guts of the sampled animals. All bark scorpion specimens 253 appeared healthy at the time of collection and no investigation into possible associated disease 254 states was undertaken. The eight polyomavirus genomes sampled from these species represent 255 three distinct species. Genomes representing species 1 and 2 were isolated from two scorpions 256 from both the liver and gut, whereas examples of species 3 were recovered from one scorpion, 257 but from both the liver and gut. This is reminiscent of the situation for more carefully studied 258 placental mammals, which typically harbor chronic co-infections with multiple polyomavirus 259 species. While it is highly likely that the viruses recovered from the liver infect scorpions, it cannot 260 be formally ruled out that the genomes recovered from the gut might reflect predation on other 261 arachnids or insects. Even if scorpions are not the true host, since they prey only on other 262 arthropods, these viruses are still likely to infect arthropods. Further investigation into the viromes 263 of scorpions and other arachnids is likely to yield evidence of a broad range of novel 264 polyomaviruses. Based on the recovery and characterization of these genomes, it appears that 265 at least one polyomavirus was circulating among the last common ancestors of arthropods and 266 chordates.

\section{Data availability}

268 The sequences have been deposited in GenBank with accession \#s MW175892-MW175899.

\section{Acknowledgements}

270 The molecular work described here was supported with startup funds from Arizona State 271 University made available to AV. This work was funded in part by the NIH Intramural Research 272 Program, with support from the $\mathrm{NCl}$ Center for Cancer Research awarded to CB.

\section{Figure legends and table text}

274 Figure 1: A. Complete genome maximum likelihood (ML) phylogenetic tree of nucleotide 275 sequences identified in this study compared with the original WGS-assembled bark scorpion 
276 polyomavirus genome (5). Tissue sources of the recovered viral genomes are denoted by

277 symbols. B. Genome-wide percentage pairwise identity comparisons between the scorpion

278 polyomaviruses. C. Genome organization of the scorpion polyomaviruses with color coded-open

279 reading frames.

280 Figure 2: Percentage pairwise identities of four major polyomavirus ORFs. Nucleotide and 281 amino acid comparisons are shown. The sources of the recovered viral genomes are denoted 282 by symbols.

283 Figure 3: Mid-point rooted maximum likelihood phylogenetic tree of the LT sequences of known 284 polyomaviruses, bandicoot papillomatosis carcinomatosis virus and those identified in this 285 study. The core fish polyomavirus LTs are highlighted with purple clades, the unusual bat 286 derived polyomavirus LTs are highlighted with orange clade. In the case of the scorpion 287 polyomavirus LTs, the symbols represent the source.

288 Figure 4: Summary of the recombination analysis of the scorpion polyomaviruses. The two 289 recombination events detected are in the LT coding region (see 1 and 2 above the genome 290 representation). The methods used to detect recombination are RDP (R) GENCONV (G), 291 BOOTSCAN (B), MAXCHI (M), CHIMERA (C), SISCAN (S) and 3SEQ (T) with the methods with 292 the lowest $p$-value being underlined.

293 Table 1: Primer details for each recovered scorpion polyomavirus

\section{References}

296 1. Lourenço WR. 2018. The evolution and distribution of noxious species of scorpions (Arachnida: Scorpiones). J Venom Anim Toxins Incl Trop Dis 24:1.

3. Webber MM, Gibbs AG, Rodríguez-Robles JA. 2015. Hot and not-so-hot females:

4. Bibbs CS, Bengston SE, Gouge DH. 2014. Activity trends and movement distances in the Arizona bark scorpion (Scorpiones: Buthidae). Environ Entomol 43:1613-1620. 

Evolutionary History of Polyomaviruses. PLoS Pathog 12:e1005574.

6. Van Doorslaer K, Kraberger S, Austin C, Farkas K, Bergeman M, Paunil E, Davison W, Varsani A. 2018. Fish polyomaviruses belong to two distinct evolutionary lineages. $J$ Gen Virol 99:567-573.

7. An P, Sáenz Robles MT, Pipas JM. 2012. Large T antigens of polyomaviruses: amazing

8. Moens U, Calvignac-Spencer S, Lauber C, Ramqvist T, Feltkamp MCW, Daugherty MD,

9. Polyomaviridae Study Group of the International Committee on Taxonomy of V,

10. López-Bueno A, Mavian C, Labella AM, Castro D, Borrego JJ, Alcami A, Alejo A. 2016.

11. Dayaram A, Galatowitsch ML, Argüello-Astorga GR, van Bysterveldt K, Kraberger S, Stainton D, Harding JS, Roumagnac P, Martin DP, Lefeuvre P, Varsani A. 2016. Diverse circular replication-associated protein encoding viruses circulating in invertebrates within a lake ecosystem. Infect Genet Evol 39:304-316.

12. Bankevich A, Nurk S, Antipov D, Gurevich AA, Dvorkin M, Kulikov AS, Lesin VM, Nikolenko SI, Pham S, Prjibelski AD, Pyshkin AV, Sirotkin AV, Vyahhi N, Tesler G, Alekseyev MA, Pevzner PA. 2012. SPAdes: a new genome assembly algorithm and its applications to single-cell sequencing. J Comput Biol 19:455-477.

14. Rosario K, Mettel KA, Benner BE, Johnson R, Scott C, Yusseff-Vanegas SZ, Baker

13. Altschul SF, Gish W, Miller W, Myers EW, Lipman DJ. 1990. Basic local alignment search tool. J Mol Biol 215:403-410.

CCM, Cassill DL, Storer C, Varsani A, Breitbart M. 2018. Virus discovery in all three major lineages of terrestrial arthropods highlights the diversity of single-stranded DNA viruses associated with invertebrates. PeerJ 6:e5761. 
15. Katoh K, Misawa K, Kuma K-I, Miyata T. 2002. MAFFT: a novel method for rapid multiple sequence alignment based on fast Fourier transform. Nucleic Acids Res 30:3059-3066.

16. Guindon S, Dufayard J-F, Lefort V, Anisimova M, Hordijk W, Gascuel O. 2010. New

17. Darriba D, Taboada GL, Doallo R, Posada D. 2011. ProtTest 3: fast selection of best-fit

18. Stöver BC, Müller KF. 2010. TreeGraph 2: combining and visualizing evidence from

19. Letunic I, Bork P. 2019. Interactive Tree Of Life (iTOL) v4: recent updates and new

20. Muhire BM, Varsani A, Martin DP. 2014. SDT: a virus classification tool based on

21. Martin DP, Varsani A, Roumagnac P, Botha G, Maslamoney S, Schwab T, Kelz Z,

22. Gabler F, Nam S-Z, Till S, Mirdita M, Steinegger M, Söding J, Lupas AN, Alva V. 2020.

23. Zimmermann L, Stephens A, Nam S-Z, Rau D, Kübler J, Lozajic M, Gabler F, Söding J,

24. Carter JJ, Daugherty MD, Qi X, Bheda-Malge A, Wipf GC, Robinson K, Roman A, Malik

\section{Johne R, Paul G, Enderlein D,} HS, Galloway DA. 2013. Identification of an overprinting gene in Merkel cell polyomavirus provides evolutionary insight into the birth of viral genes. Proc Natl Acad Sci U S A 110:12744-12749. predictions by ensembles of neural networks. Proteomics 4:1626-1632. 
371 27. Bennett MD, Woolford L, Stevens H, Van Ranst M, Oldfield T, Slaven M, O'Hara AJ,

372 Warren KS, Nicholls PK. 2008. Genomic characterization of a novel virus found in

373 papillomatous lesions from a southern brown bandicoot (Isoodon obesulus) in Western

$374 \quad$ Australia. Virology 376:173-82.

375 28. Woolford L, Rector A, Van Ranst M, Ducki A, Bennett MD, Nicholls PK, Warren KS,

376 Swan RA, Wilcox GE, O'Hara AJ. 2007. A novel virus detected in papillomas and

377 carcinomas of the endangered western barred bandicoot (Perameles bougainville)

378 exhibits genomic features of both the Papillomaviridae and Polyomaviridae. J Virol

$379 \quad 81: 13280-13290$. 
bioRxiv preprint doi: https://doi.org/10.1101/2021.05.12.443864; this version posted May 12, 2021. The copyright holder for this preprint (which was not certified by peer review) is the author/funder. This article is a US Government work. It is not subject to copyright under 17 USC 105 and is also made available for use under a CCO license.

A

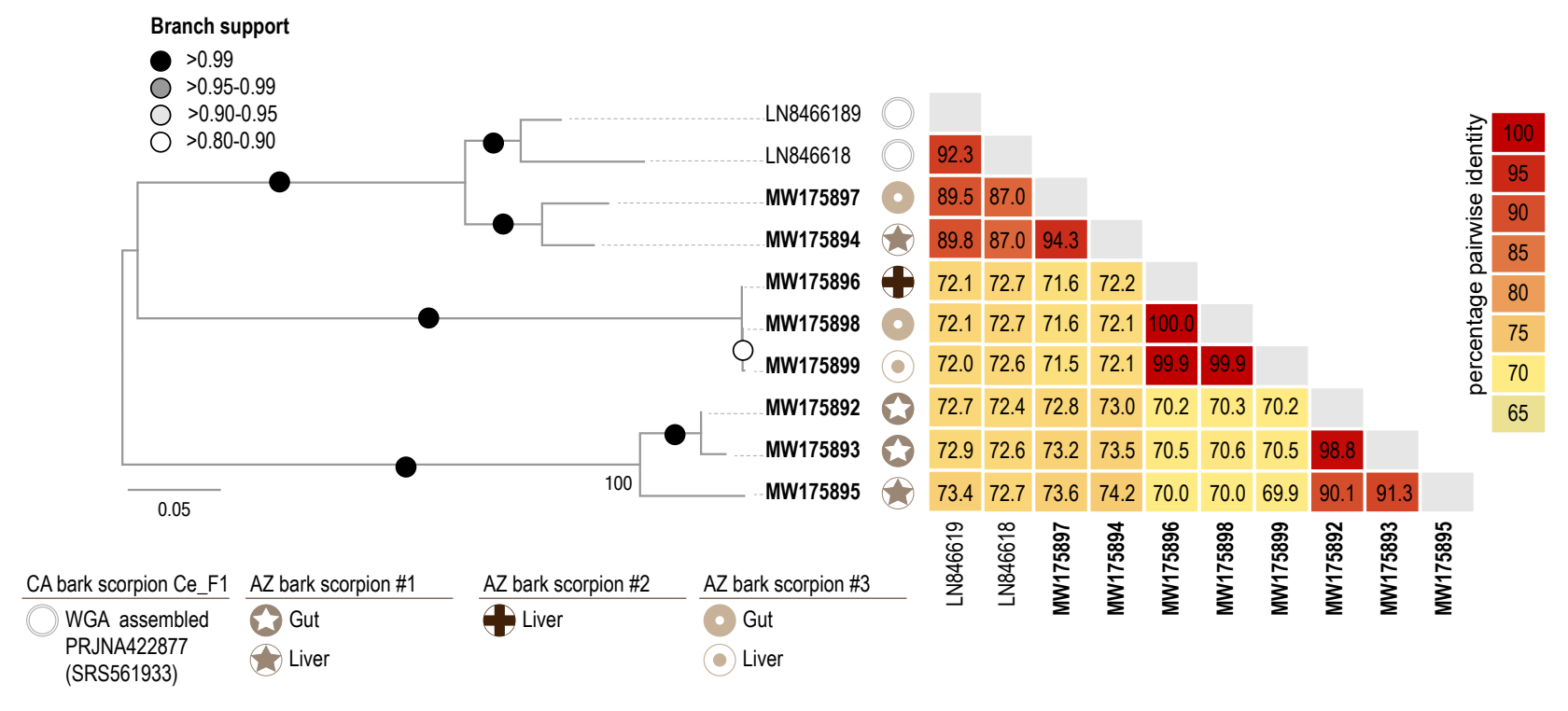

B

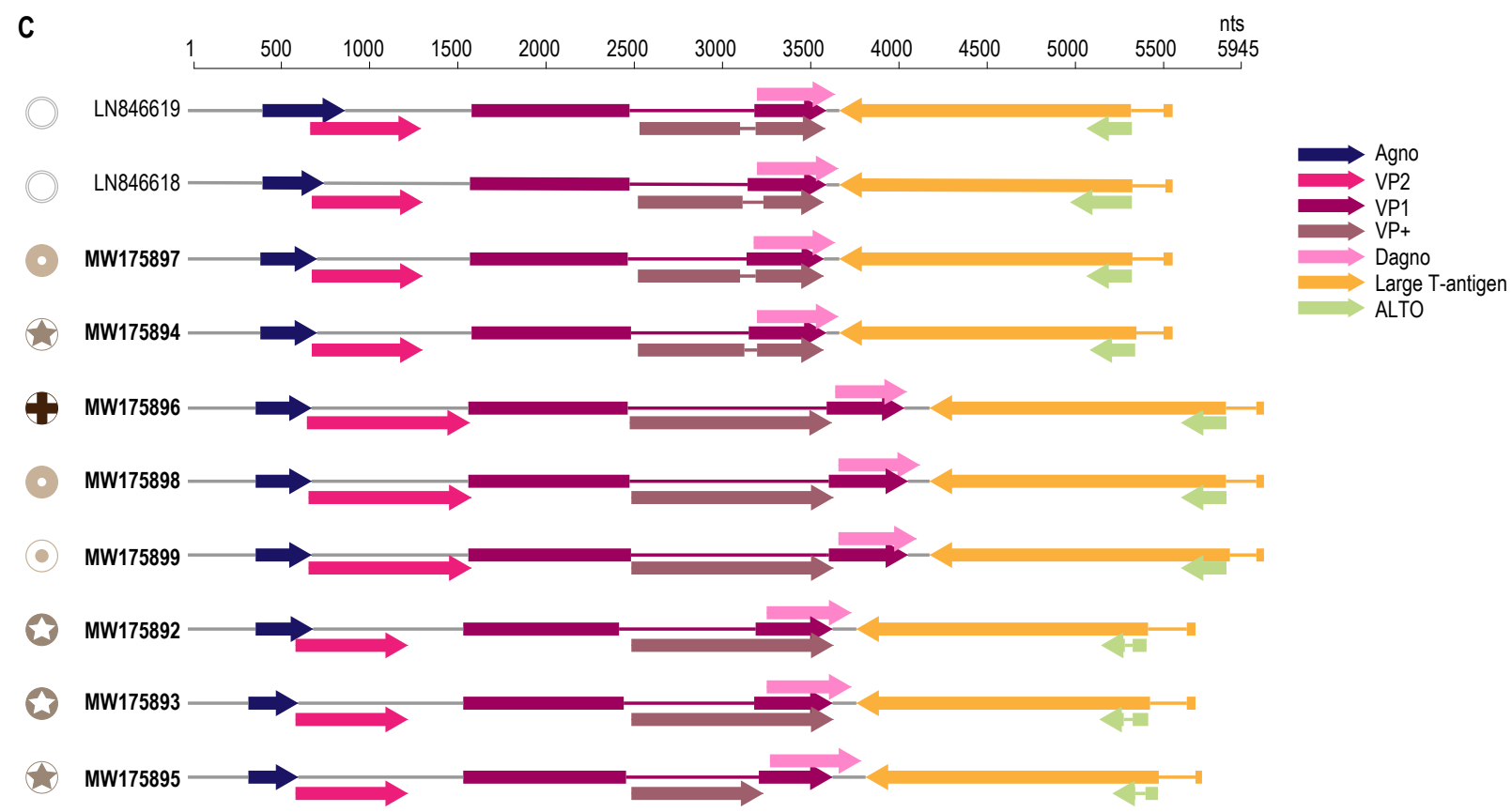

Figure 1: A. Complete genome maximum likelihood (ML) phylogenetic tree of nucleotide sequences identified in this study compared with the original WGS-assembled bark scorpion polyomavirus genome (5). Tissue sources of the recovered viral genomes are denoted by symbols. B. Genome-wide percentage pairwise identity comparisons between the scorpion polyomaviruses. C. Genome organization of the scorpion polyomaviruses with color coded-open reading frames. 
bioRxiv preprint doi: https://doi.org/10.1101/2021.05.12.443864; this version posted May 12, 2021. The copyright holder for this preprint (which

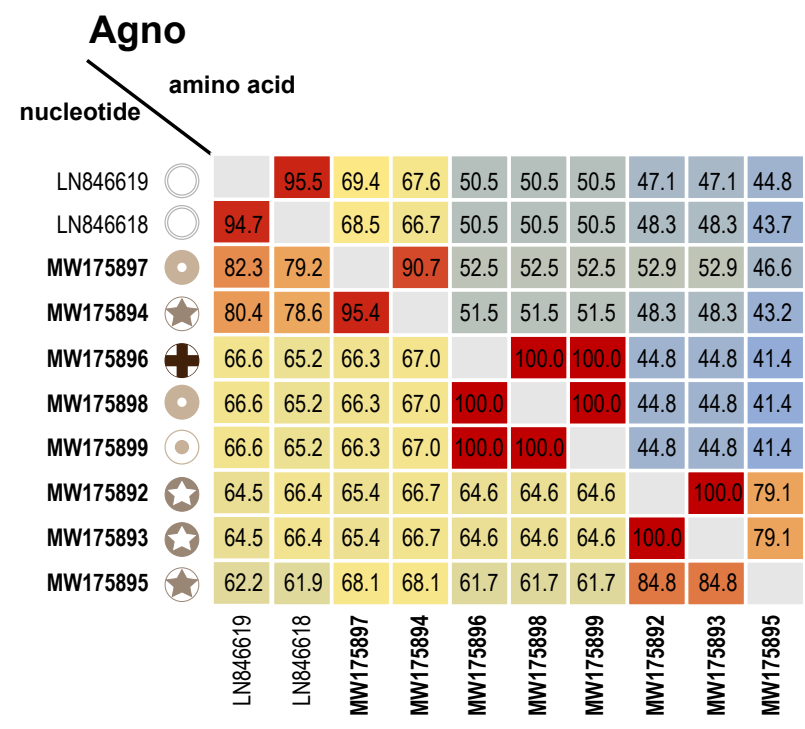

\section{VP2}

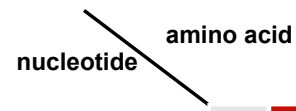

\begin{tabular}{l|l|l|l|l|l|l|l|l|l|} 
LN846619 & 98.0 & 940 & 92.5 & 50.8 & 50.8 & 50.8 & 63.9 & 63.9 & 61.3
\end{tabular}

\begin{tabular}{ll|l|l|l|l|l|l|l|l|l|}
\hline LN846618 & 93.8 & 92.5 & 92.0 & 50.3 & 50.3 & 50.3 & 64.9 & 64.9 & 61.8 \\
\hline
\end{tabular}

\begin{tabular}{ll|l|l|l|l|l|l|l|l|l|l|}
\hline MW175897 & 89.8 & 88.5 & & 97.5 & 50.3 & 50.3 & 50.3 & 61.3 & 61.3 & 61.3 \\
\hline
\end{tabular}

\begin{tabular}{l|l|l|l|l|l|l|l|l|l|l|} 
MW175894 & 90.7 & 89.0 & 95.2 & & 51.8 & 51.8 & 51.8 & 61.8 & 61.8 & 60.7 \\
\hline
\end{tabular}

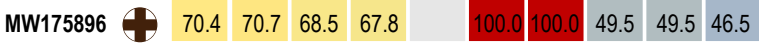

\begin{tabular}{ll|l|l|l|l|l|l|l|l|l|l|} 
MW175898 70.4 & 70.7 & 68.5 & 67.8 & 100.0 & & 100.0 & 49.5 & 49.5 & 46.5 \\
\hline
\end{tabular}

\begin{tabular}{|l|l|l|l|l|l|l|l|l|l|}
\hline MW175899 & 70.4 & 70.7 & 68.5 & 67.8 & 100.0 & 100.0 & 49.5 & 49.5 & 46.5 \\
\hline
\end{tabular}

\begin{tabular}{ll|l|l|l|l|l|l|l|l|l|l|} 
MW175892 & 70.4 & 71.5 & 72.8 & 74.3 & 65.5 & 65.5 & 65.5 & & 100.0 & 94.2 \\
\hline
\end{tabular}

\begin{tabular}{l|l|l|l|l|l|l|l|l|l|l} 
MW175893 70.4 & 71.5 & 72.8 & 74.3 & 65.5 & 65.5 & 65.5 & 100.0 & 94.2 \\
\hline
\end{tabular}

\begin{tabular}{llll|l|l|l|l|l|l|} 
MW175895 & 71.6 & 70.3 & 73.0 & 73.3 & 62.7 & 62.7 & 62.7 & 90.3 & 90.3
\end{tabular}

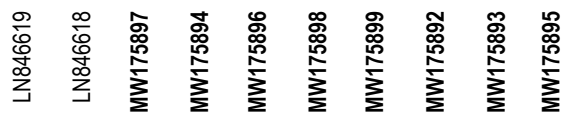

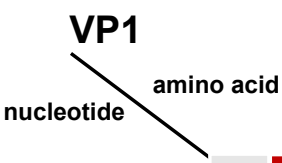

\begin{tabular}{l|l|l|l|l|l|l|l|l|l|}
\hline LN846619 & 99.8 & 91.7 & 92.5 & 63.3 & 69.6 & 69.6 & 66.8 & 67.1 & 67.5
\end{tabular}

\begin{tabular}{l|l|l|l|l|l|l|l|l|l|}
\hline LN846618 & 93.0 & 90.2 & 91.3 & 67.7 & 68.0 & 68.0 & 65.9 & 66.1 & 65.9
\end{tabular}

\begin{tabular}{l|l|l|l|l|l|l|l|l|l|l|}
\hline MW175897 & 89.2 & 89.0 & & 98.2 & 69.0 & 69.3 & 69.3 & 66.2 & 66.4 & 66.0 \\
\hline
\end{tabular}

\begin{tabular}{l|l|l|l|l|l|l|l|l|l|l|}
\hline MW175894 & 90.2 & 89.4 & 93.7 & & 69.0 & 69.3 & 69.3 & 65.7 & 66.0 & 66.4 \\
\hline
\end{tabular}

\begin{tabular}{|l|l|l|l|l|l|l|l|l|l|l|}
\hline MW175896 & 72.9 & 72.7 & 72.6 & 72.2 & & 99.8 & 99.8 & 66.7 & 67.0 & 65.4 \\
\hline
\end{tabular}

\begin{tabular}{|l|l|l|l|l|l|l|l|l|l|l|}
\hline MW175898 & 73.2 & 72.9 & 72.8 & 72.5 & 99.9 & & 100.0 & 66.8 & 67.0 & 65.5 \\
\hline
\end{tabular}

\begin{tabular}{|l|l|l|l|l|l|l|l|l|l|l|}
\hline MW175899 & 73.2 & 72.9 & 72.8 & 72.5 & 99.9 & 100.0 & 66.8 & 67.0 & 65.5 \\
\hline
\end{tabular}

\begin{tabular}{|l|l|l|l|l|l|l|l|l|l|l|}
\hline MW175892 & 70.8 & 70.5 & 70.1 & 70.3 & 70.3 & 70.3 & 70.3 & & 99.8 & 91.1 \\
\hline
\end{tabular}

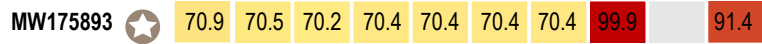

\begin{tabular}{lllll|l|l|l|l|l|l|} 
MW175895 & 72.3 & 70.9 & 72.4 & 71.8 & 70.9 & 70.8 & 70.8 & 89.9 & 89.9
\end{tabular}

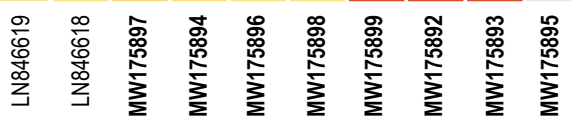

\section{Large T-antigen}

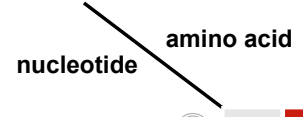

\begin{tabular}{l|l|l|l|l|l|l|l|l|l|}
\hline LN846619 @ & 96.4 & 98.6 & 98.7 & 81.1 & 81.1 & 80.7 & 82.9 & 82.9 & 82.9 \\
\hline
\end{tabular}

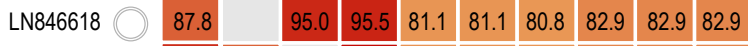

\begin{tabular}{|l|l|l|l|l|l|l|l|l|l|} 
MW175897 94.4 & 87.4 & & 98.7 & 81.3 & 81.3 & 80.9 & 83.2 & 83.2 & 83.2 \\
\hline
\end{tabular}

\begin{tabular}{l|l|l|l|l|l|l|l|l|l|l|}
\hline MW175894 & 95.1 & 86.9 & 94.2 & & 81.8 & 81.8 & 81.4 & 83.1 & 83.1 & 83.1 \\
\hline
\end{tabular}

\begin{tabular}{|l|l|l|l|l|l|l|l|l|l|l|}
\hline MW175896 & 77.0 & 77.4 & 76.4 & 77.4 & & 100.0 & 99.6 & 80.8 & 81.1 & 81.1 \\
\hline
\end{tabular}

\begin{tabular}{|l|l|l|l|l|l|l|l|l|l|l|}
\hline MW175898 & 77.0 & 77.4 & 76.4 & 77.4 & 99.9 & & 99.6 & 80.8 & 81.1 & 81.1 \\
\hline
\end{tabular}

\begin{tabular}{|l|l|l|l|l|l|l|l|l|l|l|}
\hline MW175899 & 76.7 & 77.1 & 76.1 & 77.1 & 99.7 & 99.8 & & 80.4 & 80.8 & 80.8 \\
\hline
\end{tabular}

\begin{tabular}{|l|l|l|l|l|l|l|l|l|l|l|}
\hline MW175892 & 76.9 & 76.2 & 76.4 & 77.0 & 76.2 & 76.2 & 76.0 & & 98.7 & 98.7 \\
\hline
\end{tabular}

\begin{tabular}{ll|l|l|l|l|l|l|l|l|l|l|l} 
MW175893 & 77.5 & 76.6 & 77.5 & 78.3 & 77.1 & 77.0 & 76.8 & 96.0 & 100.0 \\
\hline
\end{tabular}

\begin{tabular}{ll|l|l|l|l|l|l|l|l|} 
MW175895 77.7 & 76.7 & 77.8 & 78.1 & 76.7 & 76.7 & 76.5 & 94.3 & 98.3 \\
\hline
\end{tabular}

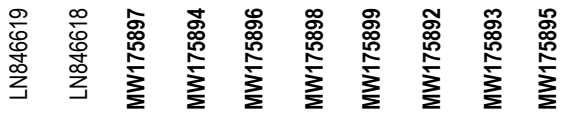

CA bark scorpion Ce_F1 AZ bark scorpion \#1

AZ bark scorpion \#2

AZ bark scorpion \#3

WGA assembled
PRJNA422877 Gut Liver

(SRS561933) Liver

Liver

percentage pairwise identity

\begin{tabular}{l|l|l|l|l|l|l|l|l|l|l|l|l|}
40 & 45 & 50 & 55 & 60 & 65 & 70 & 75 & 80 & 85 & 90 & 95 & 100
\end{tabular}

Figure 2: Percentage pairwise identities of four major polyomavirus ORFs. Nucleotide and amino acid comparisons are shown. The sources of the recovered viral genomes are denoted by symbols. 
bioRxiv preprint doi: https://doi.org/10.1101/2021.05.12.443864; this version posted May 12, 2021. The copyright holder for this preprint (which was not certified by peer review) is the author/funder. This article is a US Government work. It is not subject to copyright under 17 USC 105 and is also made available for use under a CCO license.

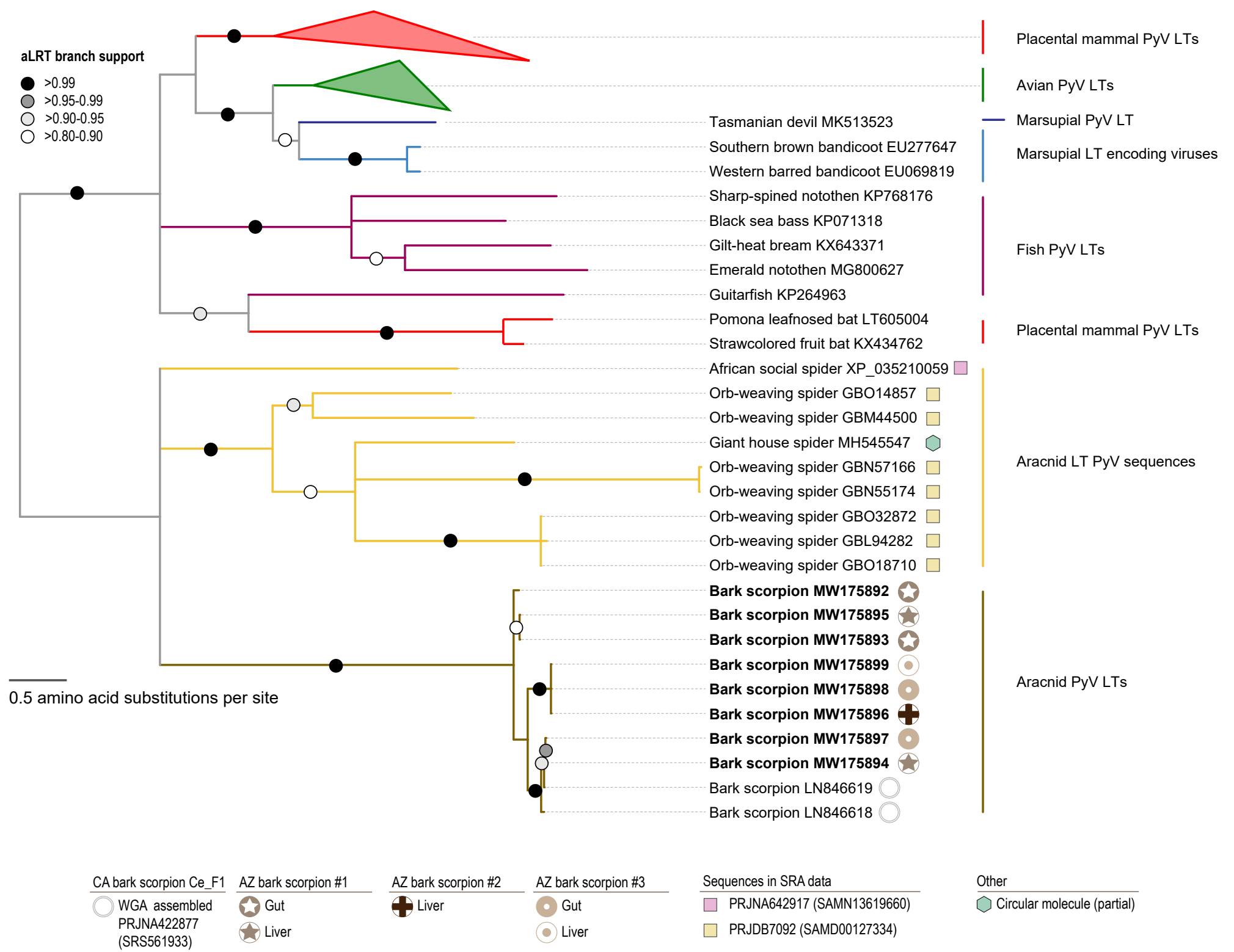

Figure 3: Mid-point rooted maximum likelihood phylogenetic tree of the LT sequences of known polyomaviruses, bandicoot papillomatosis carcinomatosis virus and those identified in this study. The core fish polyomavirus LTs are highlighted with purple clades, the unusual bat derived polyomavirus LTs are highlighted with orange clade. In the case of the scorpion polyomavirus LTs, the symbols represent the source 
bioRxiv preprint doi: https://doi.org/10.1101/2021.05.12.443864; this version posted May 12, 2021. The copyright holder for this preprint (which was not certified by peer review) is the author/funder. This article is a US Government work. It is not subject to copyright under 17 USC 105 and is also made available for use under a CCO license.

\begin{tabular}{|c|c|c|c|c|c|c|c|c|}
\hline $\begin{array}{l}\text { Recombinant } \\
\text { sequence }\end{array}$ & & $\begin{array}{l}\text { Recombinant } \\
\text { region }\end{array}$ & \multicolumn{2}{|c|}{$\begin{array}{l}\text { Recombinant } \\
\text { breakpoint }\end{array}$} & $\begin{array}{l}\text { Minor parental } \\
\text { sequence(s) }\end{array}$ & $\begin{array}{l}\text { Major parental } \\
\text { sequence }\end{array}$ & $\begin{array}{l}\text { Detection } \\
\text { method }\end{array}$ & $p$ value \\
\hline & 1 & & & & & & & \\
\hline MW175893 & $<-1$ & 1 & 4265 & 5296 & MW175895 & MW175892 & GBMCST & $8.12 \mathrm{E}-57$ \\
\hline MW175897 & $<-$ & 2 & 3932 & 3996 & $\begin{array}{l}\text { MW175895 } \\
\text { MW175892 } \\
\text { MW175893 }\end{array}$ & MW175894 & RGMC $\underline{S T}$ & 7.05E-45 \\
\hline
\end{tabular}

Figure 4: Summary of the recombination analysis of the scorpion polyomaviruses. The two recombination events detected are in the $L T$ coding region (see 1 and 2 above the genome representation). The methods used to detect recombination are RDP (R) GENCONV (G), BOOTSCAN (B), MAXCHI (M), CHIMERA (C), SISCAN (S) and 3SEQ (T) with the methods with the lowest p-value being underlined. 
bioRxiv preprint doi: https://doi.org/10.1101/2021.05.12.443864; this version posted May 12, 2021. The copyright holder for this preprint (which

was not certified by peer review) is the author/funder. This article is a US Government work. It is not subject to copyright under 17 USC 105 and is also made available for use under a CCO license.

Table 1: Primer details for each recovered scorpion polyomavirus

\begin{tabular}{ll}
\hline $\begin{array}{l}\text { Accession of full } \\
\text { genome }\end{array}$ & Primer pair used to recover for each set of viral genomes \\
\hline MW175892 & 5'-CAAATTTTCCCAACCGTAGCCTCCTTTTCCC-3' \\
MW175893 & 5'-GAAAGTCTCCGAGACCATATTGATGGTCGTATC-3' \\
\hline MW175894 & \\
MW175895 & 5'-CGAGCATACTCTTTCCGGAATTGTAATATCCGG-3' \\
MW175897 & 5'-CACATGCGTTCTCCCAATTATTCGATGCTGTG-3' \\
\hline MW175896 & \\
MW175898 & 5'-CATACGGCGGTCAAGGGCATTACCC-3' \\
MW175899 & 5'-GTCATCTTCGACGACGTCAGAGGGG-3' \\
\hline
\end{tabular}

